\title{
Minora clandestina. Le Philosophe antichrétien et autres écrits iconoclastes de l'âge classique. Sous la direction d'Alain Mothu et d'Alain Sandrier
}

\section{Peter Balats}

\section{(2) OpenEdition}

1 Journals

Édition électronique

URL : http://journals.openedition.org/studifrancesi/34668

DOI : 10.4000/studifrancesi.34668

ISSN : 2421-5856

Éditeur

Rosenberg \& Sellier

\section{Édition imprimée}

Date de publication : 1 novembre 2005

Pagination : 415-416

ISSN : 0039-2944

\section{Référence électronique}

Peter Balats, « Minora clandestina. Le Philosophe antichrétien et autres écrits iconoclastes de l'âge classique. Sous la direction d'Alain Mothu et d'Alain Sandrier », Studi Francesi [En ligne], 146 (XLIX | II) | 2005, mis en ligne le 30 novembre 2015, consulté le 19 avril 2021. URL : http://

journals.openedition.org/studifrancesi/34668; DOI : https://doi.org/10.4000/studifrancesi.34668

Ce document a été généré automatiquement le 19 avril 2021.

\section{c)}

Studi Francesi è distribuita con Licenza Creative Commons Attribuzione - Non commerciale - Non opere derivate 4.0 Internazionale. 


\title{
Minora clandestina. Le Philosophe antichrétien et autres écrits iconoclastes de l'âge classique. Sous la direction d'Alain Mothu et d'Alain Sandrier
}

\author{
Peter Balats
}

\section{RÉFÉRENCE}

Minora clandestina. Le Philosophe antichrétien et autres écrits iconoclastes de l'âge classique. Sous la direction d'ALAIN MOTHU et d'ALAIN SANDRIER, Paris, Honoré Champion, 2003, pp. 426.

1 Le présent volume constitue le seizième volumne de la collection consacrée à la production clandestine des auteurs hétérodoxes de l'Age classique. Par ailleurs, il n'est que le premier volet d'une série qui recueillera les rogatons - les minora - de la culture anti-religieuse des XVII ${ }^{\mathrm{e}}-\mathrm{XVIII}{ }^{\mathrm{e}}$ siècles. Nul n'ignore le Theophrastus Redivivus, le Traité des trois imposteurs et l'Examen de la religion (attribué à Du Marsais) qui depuis longtemps constituent un «canon» (s'il est légitime de parler d'un canon clandestin). L'objectif des éditeurs de ce volume a été de rassembler des «apocryphes», des «laissés pour compte» de cette tradition. Apparemment, il s'agit de textes très divers, tous relativement courts, peu connus et marqués de particularismes locaux. Bien que les dix textes inclus dans le volwne soient d'inspirations philosophiques très diverses (scepticisme, aristotélisme, rationalisme, "spinozisme», matérialisme ou autre), on ne peut pas leur contester une certaine unité, celle de s'ériger en critique de la religiosité traditionnelle, ainsi que celle de partager l'éthique de la libre-pensée. Ils sont précédés de 
présentations philologiques et philosophiques qui permettent au lecteur de trouver ses repères dans le monde fascinant des manuscrits clandestins.

Le premier texte intitulé Discours sur ce qu'on appelle un Philosophe chrestien est présenté par Jean-Pierre Cavaillé. Celui-ci montre que le manuscrit en question est très probablement une réponse à un ouvrage également anonyme publié en 1639: Le Philosophe Chrestien, ou les misteres de la Foy, prouvez par des raisons naturelles, un ouvrage qui tente de soutenir rationnellement, dans un esprit proche de Sebond, les mystères fondamentaux du christianisme. L'objectif de notre auteur est de démontrer que philosophe et chrétien sont deux mots absolument incompatibles, puisqu'un philosophe digne de ce nom refuse de tenir quoi que ce soit pour vrai avant de l'avoir soumis à un examen rationnel, tandis qu'un chrétien se trouve dans une position de passivité et de soumission par rapport aux dogmes de la religion. Le Dieu véritable est un Dieu à la mesure des philosophes, puisque ceux-ci peuvent presque arriver à un état de quiétude et de jouissance comparable â celui de leur Maître. L'objectif de ce texte est de réhabiliter le plaisir en délivrant l'homme de ses scrupules chrétiens - la théologie hédoniste de notre auteur s'exprime dans la pensée selon laquelle plus on obéit au principe de plaisir présent dans la nature, plus on se conforme au divin: le bordel et l'adultère ont également leur place dans l'économie rationnelle des plaisirs.

3 Le second texte, un petit écrit du grand Fontenelle, De la diversité des religions, maintes fois recopié tout au long du XVIII siècle, traite d'un sujet emprunté à Pierre Charron et à La Mothe Le Vayer: les païens - tous ceux qui n'ont jamais entendu parler du Christ seront-ils tous damnés, ou bien mériteront-ils le salut? N'existe-t-il qu'un seul chemin de la vérité? Dans ce discours académique, Fontenelle, qui fait semblant de porter les couleurs de l'orthodoxie, présente à ses lecteurs la vieille aporie de la pensée libertine: si Dieu condamne à la damnation éternelle une foule de païens vertueux et de sauvages, il est injuste; par contre, si Jésus n'est pas la seule voie du salut, la foi chrétienne (et catholique) perd son statut exceptionnel. Il n'y a qu'une seule solution à cette aporie redoutable: l'ignorance de Dieu, qui ne peut résulter que du mépris ou de l'autoaveuglement volontaire, est forcément coupable - Fontenelle, expert de la stratégie libertine, feint ironiquement d'adopter cette position, tout en démontrant ses faiblesses. Cet auteur figure d'ailleurs dans le volume avec un autre texte, intitulé Lettre sur la résurrection des corps, présenté également par Alain Mothu. Ce «badinage doucement irrévérencieux», très lu et beaucoup copié, insiste sur le manque d'espace et de matière le jour de la Résurrection. A première vue, on pourrait croire au badinage quand on lit dans la Lettre à Madame que «si les hommes marchent sur deux pieds et debout, c'est uniquement par habitude» et que l'homme est par nature un animal quadrupède. En vérité, il s'agit de la reprise d'une vieille hypothèse libertine selon laquelle l'homme n'est pas un être vertical dont les yeux seraient fixés au ciel, mais plutôt un être d'origine basse, éduquée a posteriori à sa posture actuelle.

4 Placé entre les deux textes fonteneliens, nous trouvons un poème de 74 vers, amusant et populaire, intitulé La Moysade ou L'incrédule (également présenté par Alain Mothu) qui reprend un vieux topos de la tradition libertine, notamment celui de l'imposture du grand législateur-prophète des Hébreux.

5 Afin d'illustrer l'hétérogénéité de cette culture clandestine, les éditeurs du volume ont décidé d'y intégrer deux textes inspirés d'un néo-platonisme hermétique (ou hermétisant) qui se réfère - de manière assez étrange - à la philosophie spinozienne. Les Sentiments des philosophes sur la nature de l'âme composés par Benoît de Maillet et 
présentés par Gianluca Mori sont imprégnés d'un vitalisme paracelsien qui relève plus de la tradition alchimique que du rationalisme de Spinoza. Le texte intitulé Essais de quelques idées sur Dieu est présenté par Alain Mothu (qui le qualifie de «théosophie matérialiste»). Les deux présentateurs, surtout Alain Mothu font preuve d'une érudition impressionnante - et quelque peu étonnante pour un rationaliste laïc - dans le domaine de la littérature occulto-hermético-théosophique.

6 Anthony McKenna s'est chargé de l'établissement et de la présentation de trois textes. Le premier, la version française d'un plaidoyer composé dans un contexte britannique en faveur des anti-trinitaires, partisans d'un christianisme sans mystère (pour reprendre le titre d'un ouvrage de John Toland) illustre - et ceci est un trait caractéristique de la littérature dite clandestine - l'infidélité de quelques traducteurs par rapport au texte qui leur sert de point de départ: McKenna montre que la version française de l'Infaillibilité du jugement humain supprime les réflexions de William Lyons sur la Trinité pour ne retenir que la revendication des droits de la raison humaine. Et même en restant fidèle au sens de l'original, un texte traduit, transposé d'un contexte national et religieux dans un autre, devra être interprété dans une grille de lecture toute différente. La lettre sur les difficultés de ceux qui s'appliquent à l'étude de l'Ecriture, composée par un évêque anglican en faveur de la tolérance civile devient, en traduction française, une arme efficace dans le combat «philosophique» contre l'église catholique. Le troisième texte présenté par McKenna, la Censure du Symbole des Apôtres, relève de la parodie pure et simple: l'auteur anonyme transforme un pamphlet jésuite, composé en protestation contre la censure jugée trop favorable aux jansénistes, en texte «philosophique» revendiquant la liberté de l'expression. 